\title{
Diagnóstico y tratamiento psicosocial del tabaquismo
}

\author{
SERGIO BELLO S.*, ALVARO FLORES C.**, \\ MAGDALENA BELLO S.*** y HAYDÉE CHAMORRO R.******
}

\section{Smoking cessation: Diagnosis and psychosocial intervention}

Tobacco dependence is an addictive chronic disease, characterized by its trend to the recurrence, in which the relapses are part of the process of smoking cessation. It is recommended a complete clinical evaluation, including the application of certain tests that can allow level of dependence, motivation and psychiatric comorbidities evaluation. The current treatment of smoking has two pillars: psycho-social intervention and pharmacological therapy. The current interventions are based on two theoretical models that try to understand changes of smoking behavior: The Stages of Change and PRIME Theory. Brief intervention is a strategy internationally approved because of its population impact on smoking cessation. The methodology used is named "5A's": Ask, Advise, Asses, Assist and Arrange follow-up. For not motivated patients at the intervention time it can be used the "5R's" methodology: Relevance, Risks, Rewards, Roadblocks and Repetition. The actual approach used in smokers management, is Motivational Interview, which tries to produce the behavioral change from inside and not imposing it. Its four tools are: express empathy, develop discrepancy, roll with resistance and support self efficacy. The useful psychosocial strategies, in which exists consensus, are: 1) Give practical counseling of problem solving and skills training to face risky situations; 2) Intra-treatment support, encouraging attempts of smoking cessation and communicate caring and concern.

Key words: Smoking, tobacco dependence, brief intervention, stages of change, PRIME theory, motivational interviewing, psychosocial intervention.

\section{Resumen}

El tabaquismo es una enfermedad crónica adictiva, caracterizada por su tendencia a la recurrencia, en que las recaídas son parte del proceso de dejar de fumar. Se recomienda una evaluación clínica completa de los fumadores, incluyendo la aplicación de algunos tests que permitan evaluar nivel de adicción, motivación y comorbilidad psiquiátrica. El tratamiento actual del tabaquismo tiene dos pilares: intervención psicosocial y terapia farmacológica. Las intervenciones actuales, se basan en dos modelos teóricos que intentan comprender los cambios de la conducta de fumar de las personas: las Etapas de Cambio y la Teoría PRIME. La Consejería Breve es una estrategia aprobada internacionalmente por su impacto poblacional en dejar de fumar. La metodología que utiliza se denomina las 5 A: Averiguar, Aconsejar, Acordar, Ayudar y Acompañar. Para los pacientes no motivados al momento de la intervención se utiliza la metodología de las 5 R: Relevancia, Riesgos, Recompensas, Resistencias y Repetición. El enfoque actualmente utilizado en el manejo de los fumadores, es la Entrevista Motivacional, que intenta producir el cambio conductual desde dentro y no imponiéndolo. Sus cuatro herramientas son: expresar empatía, desarrollar las discrepancias, lidiar con las resistencias y mejorar la autoeficacia. Hay consenso en que las estrategias psicosociales útiles son: 1) la entrega de consejos prácticos para resolución de problemas y desarrollo de habilidades para enfrentar situaciones de riesgo; 2) el apoyo intratratamiento, incentivando los intentos por dejar de fumar y trasmitiendo preocupación y apoyo.

Palabras clave: tabaquismo, intervención breve, etapas de cambio, teoría PRIME, entrevista motivacional, tratamiento psicosocial.

\footnotetext{
* Instituto Nacional del Tórax.

** Médico General de Zona Hospital de Achao.

*** Médico General de Zona Consultorio de Dalcahue.

**** Psicóloga.
} 


\section{Introducción}

El tabaquismo es la principal causa de diversas enfermedades respiratorias y uno de los más importantes factores de riesgo de enfermedades cardiovasculares, cáncer y de muchas otras patologías ${ }^{1}$. En todo el mundo mueren más de 4 millones de fumadores al año y en nuestro país el consumo de tabaco es responsable de más de 15.000 muertes anuales ${ }^{2}$, lo que significa un promedio de 41 muertes diarias.

Ante estos hechos, existe una preocupación mundial para controlar esta verdadera epidemia. La última iniciativa lanzada por la Organización Mundial de la Salud (OMS) durante el año 2008, es la estrategia MPOWER ${ }^{13}$. Los seis puntos de la estrategia MPOWER consisten en:

- Monitoring: Vigilar el consumo de tabaco y las medidas de prevención.

- Protecting: Proteger a la población de la exposición al humo de tabaco.

- Offering: Ofrecer ayuda para el abandono del consumo de tabaco.

- Warning: Advertir de los peligros del tabaco.

- Enforcing: Hacer cumplir las prohibiciones sobre publicidad, promoción y patrocinio.

- Raising: Aumentar los impuestos al tabaco.

En este contexto, las ayudas para dejar de fumar aparecen como uno de los pilares para el control del tabaquismo, donde los profesionales del equipo de salud tienen una gran responsabilidad.

En la actualidad existe consenso que las intervenciones de cesación del tabaquismo deben tener dos componentes: tratamiento psico-social y tratamiento farmacológico ${ }^{4-6}$. Ambos componentes deberían incluirse en el apoyo a cualquier persona que solicite ayuda para dejar de fumar.

En este artículo revisaremos el diagnóstico del tabaquismo y las terapias psico-sociales utilizadas en la actualidad. El otro componente de la terapia, los fármacos, fueron revisados recientemente ${ }^{7}$.

\section{Diagnóstico de tabaquismo y evaluación inicial del fumador}

Actualmente se considera que el tabaquismo es una enfermedad crónica sistémica que pertenece al grupo de las adicciones, clasificada como trastorno mental en el Manual diagnóstico y estadístico de los trastornos mentales (DSM IV) y en la Clasificación Internacional de Enfermedades (CIE-10) de la OMS, con criterios diagnósticos similares ${ }^{8,9}$. Al caracterizar el tabaquis- mo como enfermedad crónica, se reconoce que la mayoría de los consumidores de tabaco persisten en su consumo por muchos años y típicamente pasan por períodos de remisión y recaídas. Si bien no todos los fumadores son adictos, la mayoría de los fumadores diarios sí lo son, en los cuales es de la máxima importancia que dejen de fumar, ya que son los más expuestos a los graves daños que produce el consumo de tabaco.

El concepto de dependencia es muy importante para los médicos clínicos, ya que deben entender que las recaídas son parte del proceso y que se requiere de apoyos reiterados para lograr la abstinencia ${ }^{10}$.

Las manifestaciones clínicas dependen de las características individuales de personalidad de cada individuo, así como de las circunstancias socio-culturales que lo rodean. El principal síntoma, en los fumadores dependientes, es la necesidad imperiosa o compulsiva de volver a consumir la sustancia para experimentar la recompensa que produce y para evitar el síndrome de abstinencia.

Es recomendable hacer una evaluación general del fumador, con una completa anamnesis y examen físico. Se debe poner especial énfasis en la medición de la presión arterial, del pulso y del peso, parámetros que pueden modificarse durante el proceso de abandono del tabaco.

Dentro de los exámenes generales habrá que solicitar hemograma, perfil bioquímico (para descartar otros factores de riesgo cardiovascular asociados como hiperglicemia, hiperuricemia y dislipidemia), y en determinados casos, radiografía de tórax y espirometría.

Al ser el tabaquismo una enfermedad adictiva, se hace necesario, en cada paciente, caracterizar y objetivar la dependencia y su severidad, tanto en sus aspectos físicos como psicosociales.

En cuanto a la dependencia física ésta puede ser clasificada como leve, moderada o severa utilizando el test de Fagerström, ampliamente usado y validado para este fin ${ }^{11-13}$ (Tabla 1). Las preguntas claves son la cantidad de cigarrillos fumados al día y el tiempo que transcurre entre que la persona se levanta y fuma su primer cigarrillo.

En relación a la dependencia psicosocial, ésta puede ser valorada utilizando el test "Por qué fuma usted" (Tabla 2) ${ }^{14,15}$, que permite evaluar el rol que juega el consumo de tabaco en cada fumador en los aspectos de estimulación, manipulación, placer, manejo de la tensión, adicción, automatismo y entorno social. Este tipo de dependencia se debe diagnosticar y tra- 
Tabla 1. Test de Fagerström de dependencia a la nicotina

\begin{tabular}{|c|c|c|c|}
\hline & Pregunta & Respuesta & Puntaje \\
\hline \multirow[t]{4}{*}{ 1.- } & \multirow[t]{4}{*}{ ¿Cuántos cigarrillos fuma al día? } & 10 o menos & 0 \\
\hline & & 11 a 20 & 1 \\
\hline & & 21 a 30 & 2 \\
\hline & & 31 o más & 3 \\
\hline \multirow[t]{4}{*}{ 2.- } & \multirow{4}{*}{$\begin{array}{l}\text { ¿Cuánto tiempo pasa, desde que se levanta hasta } \\
\text { que fuma su primer cigarrillo? }\end{array}$} & Menos de 5 minutos & 3 \\
\hline & & 6 a 30 minutos & 2 \\
\hline & & 31 a 60 minutos & 1 \\
\hline & & más de 60 minutos & 0 \\
\hline \multirow[t]{2}{*}{ 3.- } & \multirow[t]{2}{*}{ ¿Fuma más en las mañanas? } & SI & 1 \\
\hline & & NO & 0 \\
\hline \multirow[t]{2}{*}{ 4.- } & \multirow{2}{*}{$\begin{array}{l}\text { ¿Tiene dificultad para no fumar en lugares donde } \\
\text { esta prohibido? }\end{array}$} & SI & 1 \\
\hline & & NO & 0 \\
\hline \multirow{2}{*}{ 5.- } & \multirow{2}{*}{ ¿A qué cigarrillo le costaría más renunciar? } & El primero del día & 1 \\
\hline & & Cualquier otro & 0 \\
\hline \multirow[t]{3}{*}{ 6.- } & \multirow{3}{*}{$\begin{array}{l}\text { ¿Fuma cuando no se encuentra bien o cuando está } \\
\text { enfermo en cama? }\end{array}$} & SI & 1 \\
\hline & & NO & 0 \\
\hline & & Puntaje Total & \\
\hline \multirow[t]{3}{*}{ Puntaje: } & Dependencia baja & & \\
\hline & Dependencia moderada. & & \\
\hline & Dependencia alta. & & \\
\hline
\end{tabular}

tar adecuadamente, ya que juega un rol importante en el fumador, quien está acostumbrado a enfrentar múltiples situaciones de su vida diaria con la ayuda de un cigarrillo: situaciones complicadas y estresantes, relaciones interpersonales o situaciones sociales (aperitivo, café, etc).

Otra evaluación que se puede incluir, es el test de Goldberg (Tabla 3), una herramienta útil para evaluar el estado psico-emocional del fumador $^{16}$, que nos permite decidir si está o no en condiciones para afrontar una terapia o debe, primero, realizar un adecuado tratamiento de su estado depresivo o ansioso.

Es muy importante tener presente que existen variables que podrían predecir el éxito o fracaso del tratamiento ${ }^{17-18}$, las cuales se deben evaluar en todo fumador. Las variables que aumentan la tasa de abstinencia son: la disposición a suspender el tabaco, el grado de autoestima, el contar con una adecuada red de apoyo y el grado de motivación, que podemos objetivar a través del test de Richmond ${ }^{19}$ (Tabla 4 ). Las variables que disminuyen las tasas de abstinencia son: alta dependencia a la nicotina, comorbilidad psiquiátrica y abuso de sustancias, niveles elevados de estrés y exposición a otros fumadores.

\section{Exámenes complementarios específicos:}

Hay dos exámenes factibles de realizar, pero que son prescindibles en la práctica clínica habitual. Estos son la medición de monóxido de carbono $(\mathrm{CO})$ en el aire espirado y de cotinina en sangre $\mathrm{u}$ orina.

Medición de $\mathrm{CO}$ en el aire espirado: El CO es uno de los constituyentes del humo de tabaco, que se puede medir a través de un cooxímetro. El CO tiene una vida media corta, entre 2 y 5 horas. La determinación de CO se utiliza para validar la afirmación verbal del fumador sobre su abstinencia y como factor motivador o de refuerzo positivo para el paciente, al poder comprobar el descenso de su concentración tras la suspensión del consumo de tabaco. Su utilidad para aumentar la tasa de abstinencia a largo plazo está actualmente en discusión ${ }^{10}$.

Determinación de cotinina: La cotinina es un metabolito de la nicotina que tiene una vida media prolongada (11 a 37 horas) y su medición se puede hacer en orina, saliva y sangre. Se utiliza principalmente para la validación de estudios clínicos y en pacientes hospitalizados altamente adictos, para titular las dosis requeridas de terapia de reemplazo de nicotina ${ }^{20}$.

Tanto la valoración conductual como los otros componentes exploratorios de la historia clínica de tabaquismo pueden ser más amplios. En la práctica clínica, el arte está en saber adaptar el tiempo disponible a las necesidades de los enfermos; aunque "lo mejor es enemigo de lo bueno", el cambio de una conducta adictiva requiere esfuerzo y dedicación, sobre todo por parte del paciente, pero también por parte del profesional de la salud ${ }^{21}$. 
Tabla 2. ¿Por qué fuma usted?

Al lado de las siguientes frases escriba el número que mejor refleje su propia experiencia.

\begin{tabular}{|c|c|c|c|}
\hline $1=$ Nunca & & $3=$ De vez en cuando & $5=$ Siempre \\
\hline & A. & Yo fumo para mantenerme activo & \\
\hline & B. & Sostener un cigarrillo en la mano forma parte del placer de fumar & \\
\hline & C. & Fumar es placentero y relajante & \\
\hline & D. & Enciendo un cigarrillo cuando me siento enojado con algo / alguien & \\
\hline & E. & Cuando se me acaban los cigarrillos, es casi una tortura hasta que consigo más & \\
\hline & F. & Fumo de manera automática, sin siquiera ser consciente de ello & \\
\hline & G. & Fumo cuando otras personas están fumando a mi alrededor & \\
\hline & H. & Fumar me ayuda a pensar y concentrarme & \\
\hline & I. & Parte del placer del cigarrillo es toda la preparación para prenderlo & \\
\hline & $\mathrm{J}$. & Fumar me produce placer & \\
\hline & $\mathrm{K}$. & Cuando me siento incómodo o molesto, prendo un cigarrillo & \\
\hline & L. & Cuando no estoy fumando, tengo el cigarrillo muy presente & \\
\hline & M & He prendido un cigarrillo cuando el anterior aún está encendido en el cenicero & \\
\hline & N. & Fumo cuando me reúno con amigos y lo estoy pasando bien & \\
\hline & O. & Cuando fumo, parte del placer es mirar el humo mientras lo exhalo & \\
\hline & P. & Casi siempre deseo un cigarrillo cuando estoy a gusto y relajado & \\
\hline & Q. & Fumo cuando estoy triste y quiero pensar en otra cosa & \\
\hline & R. & Siento verdaderas ansias de fumar cuando no lo he hecho por un buen rato & \\
\hline & S. & Me ha pasado que tengo un cigarrillo en la boca sin darme cuenta & \\
\hline & $\mathrm{T}$. & Siempre fumo cuando estoy con amigos en una fiesta, en un bar, etc & \\
\hline & U. & Fumo para darme ánimo & \\
\hline
\end{tabular}

Los puntajes obtenidos se suman de la siguiente manera, para objetivar cada condicionante de la conducta fumadora:

Estimulación: $\quad \mathrm{A}+\mathrm{H}+\mathrm{U}$

Manipulación: $\quad \mathrm{B}+\mathrm{I}+\mathrm{O}$

Placer: $\quad \mathrm{C}+\mathrm{J}+\mathrm{P}$

Manejo de tensión: $\mathrm{D}+\mathrm{K}+\mathrm{Q}$

Adicción: $\quad \mathrm{E}+\mathrm{L}+\mathrm{R}$

Hábito automático: $\mathrm{F}+\mathrm{M}+\mathrm{S}$

Fumador social: $\quad \mathrm{G}+\mathrm{N}+\mathrm{T}$

La interpretación del puntaje de cada condicionante y de su peso relativo en la conducta de cada fumador, se hace en base a la siguiente escala:

$\begin{array}{ll}\text { Bajo: } & 1 \text { a } 5 \text { puntos } \\ \text { Moderado } & 6 \text { a } 10 \text { puntos } \\ \text { Alto } & 11 \text { a } 15 \text { puntos }\end{array}$

\section{Tratamiento del tabaquismo}

Existe una amplia gama de intervenciones efectivas para la cesación tabáquica, desde la simple entrega de material de autoayuda hasta los tratamientos intensivos para dejar de fumar.

La intensidad de la intervención ${ }^{10}$ está definida por el tiempo de contacto entre el terapeuta y el paciente. Las intervenciones más simples (Consejería Breve) son menos eficaces (5 a 10\% de éxito), pero logran mayor cobertura poblacional, por lo que el número absoluto de perso- nas que dejan de fumar es mayor ${ }^{22,23}$, con una buena relación costo-efectividad. Las intervenciones más intensivas, requieren más tiempo y se desarrollan en el contexto de programas específicos y especializados para dejar de fumar, siendo de mayor eficacia (20 a $35 \%$ de éxito) pero de menor alcance poblacional ${ }^{24-26}$. La relación entre cobertura e intensidad de la intervención la vemos en la Figura 1.

Las intervenciones de ayuda para dejar de fumar están basadas en ciertos modelos teóricos, que tratan de responder las preguntas del 
Tabla 3. Test de Goldberg

$\begin{array}{lrrr}\text { 1. ¿Cómo está su salud? } \quad \text { Bien } \square \quad \text { Regular } \square & \text { Mala } \square \\ \text { 2. ¿Ha dejado de trabajar o de realizar alguna actividad por motivos de salud? } & \text { Si } \square & \text { No } \square\end{array}$

A continuación quisiéramos saber si Ud. ha tenido problemas médicos y como ha estado su salud en las últimas semanas. Por favor responda todas las preguntas simplemente marcando con una cruz la respuesta que cree que corresponde a su caso. Recuerde que deseamos saber sobre las molestias presentes y recientes, no de aquellas que tuvo en el pasado.

Es importante que Ud. responda todas las preguntas.

1. ¿Recientemente ha dejado de dormir por preocupaciones?

\begin{tabular}{|c|}
\hline No en absoluto \\
\hline No más que lo habitual \\
\hline Un poco más que \\
\hline Mucho máso \\
\hline
\end{tabular}

3. ¿Ha podido concentrarse bien en lo que hace?

$$
\begin{aligned}
& \square \text { Más que lo habitual } \\
& \text { Igual que siempre } \\
& \square \text { Menos que lo habitual } \\
& \square \text { Mucho menos que lo habitual }
\end{aligned}
$$

5. ¿Ha sido capaz de enfrentar sus problemas?

$$
\begin{aligned}
& \square \text { Más que lo habitual } \\
& \text { Igual que siempre } \\
& \text { Menos que lo habitual } \\
& \text { Mucho menos que lo habitual }
\end{aligned}
$$

7. ¿Ha sentido que Ud. no puede resolver sus dificultades?

$$
\begin{aligned}
& \square \text { No en absoluto } \\
& \square \text { No más que lo habitual } \\
& \square \text { Un poco más que lo habitual } \\
& \square \text { Mucho más que lo habitual }
\end{aligned}
$$

9. ¿Ha disfrutado de sus actividades diarias?

$$
\begin{array}{ll}
\square & \text { Más que lo habitual } \\
\square & \text { Igual que siempre } \\
\square & \text { Menos que lo habitual } \\
\square & \text { Mucho menos que lo habitual }
\end{array}
$$

11.¿Ha perdido confianza en si mismo?

$$
\begin{aligned}
& \square \text { No en absoluto } \\
& \square \text { No más que lo habitual } \\
& \square \text { Un poco más que lo habitual } \\
& \square \text { Mucho más que lo habitual }
\end{aligned}
$$

\section{NOMBRE:}

\section{FECHA:}

PUNTAJE:

Puntaje: Se da 0 punto por cada respuesta marcada en las dos respuestas superiores y 1 punto en caso de marcar cualquiera de las dos inferiores. Si el puntaje alcanzado es de 5 o más puntos, se considera que la persona no está en condiciones psico-emocionales adecuadas para enfrentar la terapia. 


\section{Tabla 4. Test de Richmond}

1. ¿Le gustaría dejar de fumar si pudiera hacerlo fácilmente?
No......
0 punto
Si.......
1 punto

De acuerdo a la siguiente escala, de 0 a 3 , desde 0 en absoluto, hasta 3 muy seriamente, conteste las preguntas 2, 3 , y 4.

2. ¿Cuánto interés tiene en dejarlo?.........puntos

3. ¿Intentará dejar de fumar en las próximas cuatro semanas?.......puntos

4. ¿Cuál es la probabilidad de que dentro de los próximos 6 meses sea un no fumador? .puntos

El cálculo del puntaje final debe hacerse de acuerdo a la siguiente escala: 0 - 6: Baja motivación

7 - 8: Moderada motivación

9-10: Alta motivación

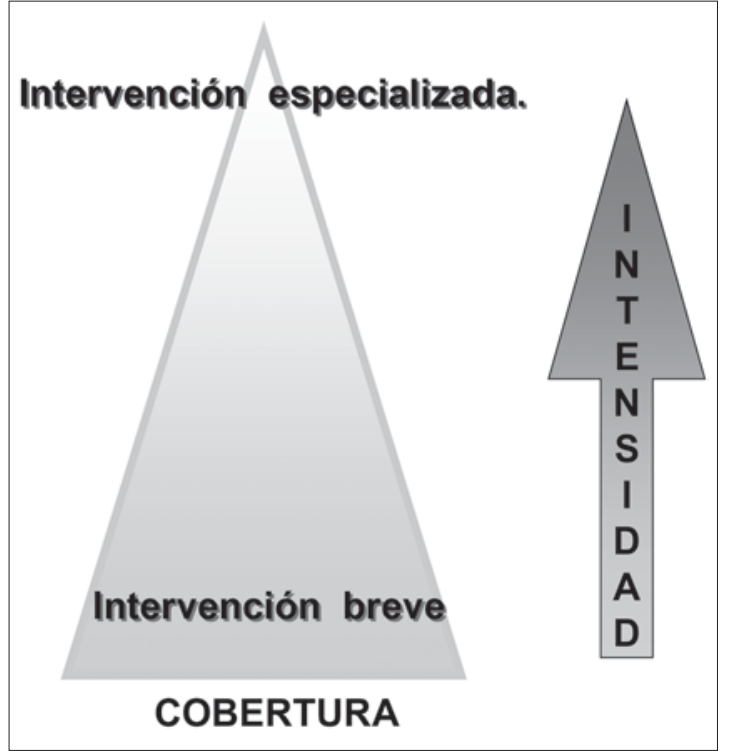

Figura 1. Tipos de intervención para dejar de fumar y su relación con cobertura e intensidad (modificado de ref. 36).

porqué y cómo cambian su conducta de fumar las personas.

En la actualidad hay dos modelos que se están utilizando ampliamente en el mundo: el modelo de las Etapas de Cambio (o teoría transteorética) de Prochaska y DiClemente ${ }^{27,28}$ que se utiliza hace más de 20 años y la teoría PRIME de Robert West ${ }^{29,30}$, de más reciente formulación y uso.

De acuerdo al modelo de etapas de cambio, dejar de fumar es un proceso en el cual existen etapas, hasta llegar a la abstinencia definitiva. Estas etapas son: precontemplación, contemplación, preparación, acción y mantención. En la etapa de precontemplación la persona ni siquiera considera la posibilidad de dejar de fumar. En la de contemplación el fumador ha pensado la posibilidad de abandonar el cigarrillo, dentro de los próximos 6 meses, pero aún está indeciso e inseguro. La etapa de preparación es aquella en que la persona visualiza dejar de fumar en los próximos 30 días y hace algo por dejar de fumar, sin abandonarlo totalmente, como por ejemplo fumar una menor cantidad diaria, empezar a fumar más tarde, no fumar dentro de la casa, etc. En la etapa de acción, el fumador deja de fumar totalmente y corresponde a los 6 primeros meses de abstinencia. Finalmente, la mantención es la etapa que va más allá de los de 6 meses de detención. En las etapas de acción y mantención son relativamente frecuentes las recaídas, por lo que se considera que éstas también forman parte del proceso, para volver a reinsertarse en las etapas de precontemplación, contemplación o preparación. El rol del profesional de la salud sería ayudar al paciente a moverse de una etapa a otra, avanzando de acuerdo a la situación particular de cada fumador.

Han surgido diversas críticas a este modelo, como por ejemplo: la arbitrariedad de los plazos, la poca importancia que le da al rol de los refuerzos y castigos en el desarrollo de hábitos difíciles de superar y al impacto inmediato de las barreras y facilitadores del cambio de conducta. Por otra parte, se han realizado intervenciones basadas en el modelo de etapas de cambio y no han probado ser más efectivas que las que no las consideran ${ }^{31}$.

Recientemente se ha elaborado otro enfoque del proceso de dejar de fumar, denominada teoría PRIME de la motivación, que señala que en toda adicción existe una fuerte motivación que lleva a una conducta desadaptativa, sobrepasando la capacidad de autocontrol.

La teoría PRIME de la motivación presenta 
un modelo de 5 niveles integrados, que interactúan entre si. Estos son: Planes, Respuestas, Impulsos/Inhibiciones, Motivos y Evaluaciones: de las iniciales de cada uno de los niveles surge el acrónimo PRIME. El nivel de los planes $(\mathbf{P})$ o reglas se basa en la capacidad humana de proyectarse. Los planes orientan la vida humana, constituyendo una estructura para las actividades cotidianas. Así, el individuo está programado para fumar en determinadas circunstancias, como después de terminar una actividad, cada vez que toma café, etc. El nivel $\mathbf{R}$ lo constituyen las respuestas reflejas a estímulos específicos: el fumador enciende el cigarrillo sin decidirlo conscientemente ni pensar en las consecuencias. En el nivel I los impulsos e inhibiciones aparecen como respuestas automáticas, ligadas a estímulos específicos, que pueden ser innatas (instintos) o aprendidas (hábitos). En el fumador, cada aspiración de un cigarrillo resulta en una rápida absorción de nicotina y consecuente elevación de dopamina, con lo que se producen asociaciones estímulo-impulsos, generando el impulso a fumar. La sensación placentera al fumar genera los motivos (M): sentimientos de deseo (placer o satisfacción anticipada) o necesidad (alivio anticipado) que son centrales en la conducta dirigida a metas. A un nivel cognitivo aparecen las evaluaciones $(\mathbf{E})$ o "creencias" que forman los fumadores acerca de los beneficios de fumar en relación al manejo del estrés, control del peso y logro de concentración, entre otros, por lo que en determinadas circunstancias desean o necesitan fumar. Según este enfoque, la conducta humana es el resultado, en cada momento, de la interacción de múltiples influencias sobre los diferentes niveles de motivación.

La cesación del tabaco necesita autocontrol y la aplicación de un plan en la fase de deseos, necesidades o impulsos competitivos. Esto requiere esfuerzo y la presencia de reglas con límites claros que faciliten superar el conflicto con otros deseos y necesidades. Los planes en forma de "reglas" organizan la conducta y protegen los intereses de largo plazo frente a las demandas inmediatas, propiciando un cambio en la identidad personal, de "fumador" a "ex fumador".

Esta teoría permite entender los mecanismos que interactúan a la base de la adicción al cigarrillo, permitiéndonos reconocer las vías de intervención para un efectivo proceso de cesación tabáquica.

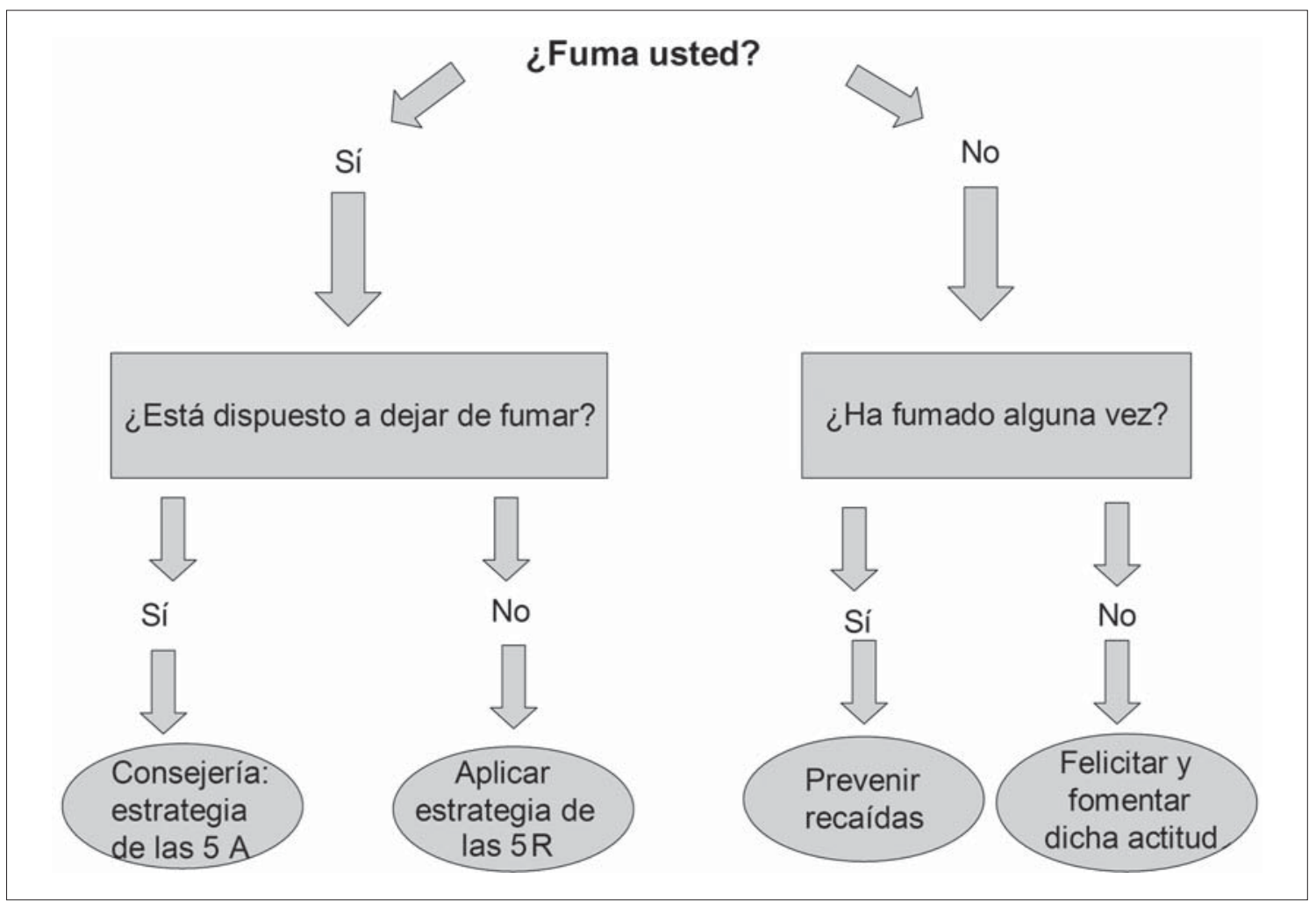

Figura 2. Algoritmo para tratar el consumo de tabaco (modificada de ref. 10). 


\section{1) Enfrentamiento clínico del tabaquismo}

A cualquier persona que consulte a un establecimiento de salud, público o privado, deberá preguntársele si consume o no tabaco, de acuerdo al algoritmo de la Figura 2. Cuando la persona está dispuesta a intentar dejar de fumar, la estrategia general recomendada es la de las $5 \mathrm{~A}$, la que se puede adaptar desde la intervención breve a la especializada, dependiendo del tiempo y capacitación del terapeuta. Cuando el fumador no quiere dejar de fumar en ese momento, se utiliza la estrategia de las $5 \mathrm{R}$. También en estos casos se puede utilizar la Entrevista Motivacional, como una estrategia de probada eficacia para motivar a aquellos fumadores sin interés por dejar de fumar. Para realizar la Entrevista Motivacional se requiere de un terapeuta entrenado, por lo que habitualmente dicha técnica es utilizada en programas especializados.

A continuación se desarrollarán las tres estrategias mencionadas.

\section{2) Consejería breve: metodología de las 5 A}

En la práctica, la mayoría de las intervenciones están basadas en una metodología, surgida en el Instituto Nacional del Cáncer de EE.UU. ${ }^{32}$ $\mathrm{y}$ aceptada por las principales guías de tratamiento del mundo ${ }^{10,33-37}$, que se denomina las 5 A, por la inicial de las 5 etapas que la constituyen: Averiguar, Aconsejar, Acordar, Ayudar y Acompañar. Consiste básicamente en estimular y promover la intención de dejar de fumar y ayudar a aquellos ya motivados en la cesación del tabaquismo. La consejería breve debiera realizarse en la consulta de cualquier profesional de la salud ${ }^{38}$, independiente del motivo de consulta. Un profesional entrenado no debiera demorarse más de dos o tres minutos en su aplicación.

Se ha utilizado la entrega de materiales de auto-ayuda junto con la consejería breve, pero no hay evidencias que demuestren un beneficio adicional. Sí existe evidencia, discreta pero significativa, de que materiales adaptados al perfil de cada persona, son más efectivos que los generales $^{39}$.

\section{Averiguar}

Se les debe preguntar a todos los pacientes por el hábito de fumar, aprovechando todas las instancias por las cuales concurren a los establecimientos de salud. Se le pregunta por el número de cigarrillos que fuma a diario y el momento del primer cigarrillo después de levantarse.

\section{Aconsejar}

Después de preguntar, se debe aconsejar al paciente que deje de fumar. El consejo debe ser claro y firme, personalizando los argumentos de convencimiento: en adolescentes, se debe insistir sobre el efecto del cigarrillo en su capacidad de ejercicio y mal rendimiento escolar; a la embarazada le preocupa su hijo y los riesgos del parto; el adulto teme más a las enfermedades que podría desarrollar por el tabaco y los posibles daños que puede causarle a los hijos y a los que lo rodean.

\section{Acordar el tipo de intervención}

Existen diferentes tipos de intervenciones, dependiendo de la etapa de cambio en que se encuentre.

a) Si el paciente no desea dejar de fumar ahora: explicitar lo perjudicial del consumo de tabaco y ofrecer apoyo para el futuro.

b) Si el paciente está inseguro: discutir sus miedos y fomentar la motivación explicando las ventajas de no fumar. Invitarlo a dejar el tabaco cuando esté listo.

c) Si el paciente está decidido: ofrecer ayuda y planear una estrategia para dejar de fumar.

\section{Ayudar}

Si el paciente está dispuesto a intentar dejar de fumar, se le debe ayudar para desarrollar un plan de acción y eventual apoyo farmacológico.

Se selecciona con el paciente una fecha para dejar de fumar, dentro de las próximas 2 a 4 semanas, la que se denomina el "Día D". No es aconsejable hacerlo en períodos de alto estrés, $\mathrm{y}$ por otra parte, es necesario considerar que no hay un tiempo ideal para dejar de fumar, pero antes es mejor que después.

Para afianzar el compromiso se sugiere la firma de un contrato-compromiso especificando la fecha acordada y donde el terapeuta también se compromete a prestar todo su apoyo al paciente.

\section{Acompañar}

En los pacientes que están en la etapa de acción, se debe programar el seguimiento. Se recomienda establecer una visita de control una a dos semanas después del "Día D". Una segunda visita de seguimiento debería acordarse un mes después de la primera, como control más alejado.

Otras recomendaciones generales que se pueden hacer, se resumen en la Tabla 5. 
Tabla 5. Recomendaciones generales para dejar de fumar

\section{Preparación}

- Recalcar la importancia de establecer una fecha para abandonar el tabaco, después de la cual la abstinencia deberá ser completa

- Aconsejar que informe a familiares y amigos sobre esta decisión

- Sugerir eliminar el material relacionado con el tabaco (ceniceros, cigarreras y encendedores)

- En caso de apoyo farmacológico, explicar su papel y posibles efectos adversos

- Discutir las dificultades con las que se podría enfrentar: síntomas de abstinencia, aumento de peso y recaídas

\section{Mantención}

- Felicitar la abstinencia completa y reforzar la motivación

- Evaluar síntomas de abstinencia y explicar que cederán con el tiempo

- Manejo de las urgencias por fumar: evitar situaciones de riesgo y distraerse con otras actividades

- Considerar metas de corto plazo. Ejemplo: "hoy no fumo"

- Evaluar el uso del tratamiento farmacológico y resolver problemas al respecto

- Insistir en que a medida que pasa el tiempo se va haciendo más fácil

- Si ha ocurrido una recaída, identificar las circunstancias en que ocurrió y revisar formas de enfrentarlas en el futuro. Si ha fumado sólo unos pocos cigarrillos, aconsejar que debe parar inmediatamente; si tuvo una recaída completa, puede ser necesario establecer un nuevo Día D

\section{3) Estrategias a utilizar cuando los pacientes no quieren dejar de fumar}

Hay pacientes que no están dispuestos a hacer un intento por dejar de fumar en ese momento. Esto puede ser porque: carecen de información acerca de los daños que produce el consumo de tabaco y de los beneficios de la abstinencia, tienen algunos miedos o preocupaciones acerca de dejar o están desmoralizados por fracasos previos ${ }^{40,41}$. Estas personas pueden responder a una estrategia denominada Entrevista Motivacional (EM) que es una intervención centrada en el fumador, que intenta ayudarle a desarrollar sus propias habilidades, con vista a estimular el cambio de conducta, ayudando a los pacientes a explorar y resolver su ambivalencia respecto al consumo de tabaco ${ }^{42}$. Hay evidencias que la EM es efectiva para incrementar los intentos por dejar de fumar en el futuro ${ }^{43,44}$.

El enfoque o espíritu de la EM es de colaboración, en que el fumador participa, por ejemplo, en la creación de su propio plan de tratamiento. También se utiliza la evocación: el terapeuta evoca en el paciente ideas y sentimientos de cambio, manteniendo siempre el respeto por su autonomía. El terapeuta no actúa como autoridad, sino que utiliza la propia experiencia del paciente. El objetivo central consiste en aumentar la motivación intrínseca del fumador, de manera que el cambio surja desde dentro, más que se imponga desde fuera ${ }^{45}$. Las cuatro herramientas de la EM son: expresar empatía, desarrollar las discrepancias, lidiar con la resistencia y apoyar la autoeficacia. Cada una de estas herra- mientas tiene estrategias específicas, que se detallan en la Tabla 6.

El contenido de las áreas que deben trabajarse en la entrevista motivacional se ha sistematizado en la Estrategia de las "5 R"10, denominada así por la inicial de los 5 puntos a considerar: Relevancia, Riesgos, Recompensas, Resistencias y Repetición.

Relevancia: Discutir con el paciente la importancia que tendría el dejar de fumar para él, por ejemplo en sus hijos, familiares o enfermedades.

Riesgos: Ayudar a que el paciente identifique los riesgos de fumar. Enfatizar que fumar cigarrillos bajos en nicotina o usar otras formas de tabaco, no eliminará estos riesgos. Ejemplos: empeoramiento de sus enfermedades, impotencia sexual, cónyuge con mayor riesgo de cáncer pulmonar y enfermedad coronaria, etc.

Recompensas: Incentivar al paciente a que reconozca los beneficios de dejar de fumar. Ejemplos: mejoría de su salud, ahorro de dinero, mejor olor personal y de la casa, hijos más sanos, etc.

Resistencias: Intentar que el paciente identifique las barreras para dejar de fumar y ofrecer ayuda para enfrentarlas. Ejemplos: síntomas de abstinencia, aumento de peso, etc.

Repetición: Se debe repetir esta estrategia cada vez que consulte un fumador no motivado.

$\mathrm{Si}$ esto se realiza en forma sistemática, se logra que muchos fumadores se motiven $\mathrm{y}$ concreticen intentos por dejar de fumar ${ }^{10}$. 
Tabla 6. Estrategias de la Entrevista Motivacional

\section{Expresar empatía}

- Usar preguntas abiertas para explorar la importancia de fumar, preocupaciones y beneficios de dejar de fumar. Ej. “QQué ocurriría si usted dejara de fumar?”

- Usar la escucha activa para manifestar comprensión, resumir y reformular frases. Ej. “Así que usted cree que fumar le ayuda a mantener su peso"

- Normalizar sentimientos y preocupaciones. Ej. "Mucha gente se preocupa sobre cómo dejar de fumar"

- Apoyar la autonomía del paciente y el derecho a rechazar el cambio. Ej. "Usted dice que no está listo para dejar el tabaco ahora. Cuando sí lo esté, podemos ayudarlo"

\section{Desarrollar discrepancia}

- Destacar la discrepancia entre la conducta actual del paciente y sus prioridades, valores o metas. Ej. "Parece que usted es muy apegado a su familia. ¿Cómo cree que afecta a sus hijos al seguir fumando?"

- Construir y profundizar en el compromiso al cambio. Ej. "Nos gustaría ayudarlo a evitar un infarto, como el que sufrió su padre"

\section{Lidiar con la resistencia}

- Retroceda y use reformulación cuando el paciente exprese resistencia. Ej. "Parece que se siente presionado para dejar de fumar"

- Pida permiso para entregar información. Ej. “¿Le gustaría saber algunas estrategias que pueden ayudarlo cuando deje de fumar?

\section{Apoyar la autoeficacia}

- Ayude al paciente a identificar y construir sobre éxitos anteriores. Ej. "Así que le fue bien la última vez que intentó dejar de fumar"

- Ofrezca opciones para lograr pequeños pasos hacia el cambio. Ej. Cambiar patrones de fumar, como no fumar en la casa

\section{4) Tratamiento psico-social}

Las estrategias psico-sociales, que mayoritariamente tienen un enfoque cognitivo-conductual, están destinadas a que el fumador reconozca su adicción, modifique sus pautas de comportamiento y desarrolle estrategias y habilidades para lograr la abstinencia, lo que significa cambiar estilos de vida, es decir, aprender a "vivir sin tabaco" $" 46$.

Las terapias cognitivo-conductuales son las más utilizadas en el tratamiento del tabaquismo. Más de 100 estudios controlados han demostrado su eficacia, por lo que son consideradas como terapia de primera línea en la Guía de Tratamiento 2008 del Servicio de Salud Pública de EE.UU. ${ }^{10}$.

Las estrategias psico-sociales se pueden desarrollar de diferentes maneras en la práctica: terapias individuales, terapias grupales, líneas telefónicas de ayuda, tratamientos a través de internet, etc. No se ha encontrado diferencia de eficacia entre la intervención individual y la grupal $^{47}$, pero esta última tiene mejor relación costo-efectividad $^{48}$. El consejo telefónico, especialmente la modalidad pro-activa, ha demostrado ser eficaz, costo-efectivo y con capacidad de gran cobertura poblacional ${ }^{49}$.

Las estrategias psico-sociales que han demostrado ser efectivas en el tratamiento del tabaquismo son ${ }^{10,21,35,36 \text { : }}$ a. Provisión de consejos prácticos: técnicas de resolución de problemas, entrenamiento para el desarrollo de habilidades y competencias:

Es un conjunto de estrategias, en el marco cognitivo-conductual, que le permiten al fumador desarrollar habilidades que contribuyen a cambiar su patrón de conducta. Los componentes más importantes de estos consejos prácticos son los siguientes:

- Reconocer situaciones de riesgo: Identificar estados internos o actividades que aumenten el riesgo de fumar. Estas situaciones son múltiples y varían en cada persona, dependiendo de la relación personal con el tabaco a lo largo de los años, por ejemplo:

- Estados de ánimo negativos y estrés

- Estar cerca de otros fumadores

- Beber alcohol

- Experimentar urgencia por fumar

- Disponibilidad de cigarrillos

- Desarrollar habilidades de enfrentamiento y resolución de problemas, con el objetivo de enfrentar las situaciones de riesgo:

- Aprender a anticiparse y evitar situaciones gatillantes

- Aprender estrategias cognitivas que reducirán ánimos negativos

- Lograr cambios de estilo de vida que reduzcan el estrés, mejoren la calidad de vida, 
y reduzcan la exposición a gatillos para fumar

- Aprender actividades cognitivas y conductuales para enfrentar la urgencia por fumar (por ejemplo, distracción de la atención, cambiar rutinas), teniendo presente que este deseo cede al cabo de unos pocos minutos.

- Adquirir habilidades de asertividad.

- Proveer información básica sobre fumar y cómo dejar de fumar exitosamente: Se trata de que el fumador adquiera una serie de conocimientos básicos sobre el tabaquismo en general y sobre su adicción en particular, para que no se deje influir por pensamientos desajustados o erróneos.

- La naturaleza adictiva de fumar.

- Cualquier cigarrillo fumado (incluso una "piteada") aumenta el riesgo de una recaída completa.

- Los síntomas de abstinencia generalmente son más intensos en las dos primeras semanas del cese tabáquico, pero pueden persistir por más tiempo. Estos síntomas incluyen ánimo negativo, urgencia por fumar y dificultad para concentrarse.

\section{b. Apoyo intra tratamiento:}

Este es un conjunto de estrategias proporcionadas por el equipo de salud, dirigidas a reforzar las motivaciones positivas del fumador, destacando las ganancias sobre las pérdidas, aumento de la autoconfianza, evitando mitos y racionalizaciones en torno al consumo (como la fantasía del control de la cantidad del consumo), prevenir problemas asociados al abandono del tabaco (aumento de peso, irritabilidad, etc.) $y$ ayudando al fumador a resolver sus ambivalencias. Las principales estrategias son.

- Estimular al paciente para hacer el intento de dejar de fumar

- Mencionar que ahora existen tratamientos efectivos para la dependencia del tabaco

- Comunicar confianza en la capacidad del paciente para dejar de fumar

- Trasmitir preocupación

- Expresar directamente preocupación y disposición para ayudarlo(a) con la frecuencia que sea necesaria.

- Preguntar sobre los miedos del paciente y ambivalencia sobre dejar de fumar

- Incentivar al paciente a hablar sobre el proceso de dejar de fumar, preguntando por:
Las razones por las que quiere dejar de fumar

- Preocupaciones sobre dejar de fumar

- Éxitos que ha conseguido el paciente.

\section{c. Apoyo social, extra-tratamiento, brindado} por la familia, entorno laboral o comunitario:

Son las acciones destinadas a conseguir colaboración y apoyo de familiares y amigos en el proceso de recuperación, por ejemplo instaurando ambientes libres de humo en el hogar y en el trabajo, estableciendo "socios" de apoyo y ayudar a otros fumadores para dejar de fumar. Sin embargo, recientemente se ha puesto en duda su efectividad ${ }^{10}$.

\section{Comentarios finales}

Las políticas públicas dirigidas al tratamiento del tabaquismo tienen un efecto relativamente pequeño sobre la prevalencia general del tabaquismo en una población, logrando reducciones de alrededor de 1 a 2 puntos porcentuales ${ }^{36}$. Sin embargo, su disponibilidad y desarrollo son muy importantes para todos los fumadores, pero en especial para aquellas personas que tienen más dificultades para dejar de fumar, y para las que ya presentan alguna patología secundaria al consumo de tabaco y que deben dejar de fumar.

En la actualidad, el mejor conocimiento de la psicopatología de la adicción al tabaco y de las motivaciones para el cambio de conducta, permite comprender mejor a los fumadores y mejorar el apoyo psico-social que se les puede brindar.

Por otra parte, herramientas como la Entrevista Motivacional y las estrategias cognitivo conductuales, han demostrado su utilidad para aumentar y potenciar la motivación y para aumentar el éxito de los que intentan dejar de fumar.

Estos avances, junto con el desarrollo de nuevos fármacos más eficaces, deben incentivar a los profesionales del equipo de salud y especialmente a los médicos a asumir un rol más activo en ofrecer apoyo y tratamiento a todo fumador que manifieste intención de dejar de fumar. Se han identificado barreras ${ }^{50}$ que en el pasado dificultaban este rol: temor a dañar la relación médico-paciente, falta de conocimientos sobre la forma de ayudar a los pacientes y creencia de que resultará inefectivo. Pero las evidencias hoy son distintas y se puede hacer mucho por ayudar a los fumadores.

Se debe aprovechar cualquier contacto del 
fumador con el sistema de atención de salud para incentivar y promover el cese del consumo de tabaco. Además es perentorio involucrar a todos los profesionales de salud para que en cada consulta hagan la intervención breve. Si el paciente no logra dejar de fumar, deberá derivarse para una intervención especializada.

Por otra parte, la capacitación para el enfrentamiento del tabaquismo debiera ser parte del currículo de pregrado de todas las carreras de la salud, con mayor extensión y profundidad de lo que se hace en la actualidad. También debería haber una preocupación más explícita y permanente de las sociedades médicas para capacitar a sus miembros en este tema, especialmente aquellos más relacionadas con los daños del consumo de tabaco, contribuyendo a disminuir las tasas de mortalidad producidas por el tabaquismo.

\section{Bibliografía}

1.- US DEPARTMENT OF HEALTH AND HUMAN SERVICES. The health consequences of smoking: A report of the Surgeon General, 2004. http://www.cdc. gov/tobacco/data_statistics/sgr/2004/index.htm Consultado el 2 de Agosto 2009.

2.- MINISTERIO DE SALUD. Departamento de Estadísticas e Información en Salud. 2009 http://deis.minsal.cl/ deis/tabaco/muertes archivos/sheet002.htm Consultado el 2 de Agosto de 2009.

3.- ORGANIZACIÓN MUNDIAL DE LA SALUD. http:// www.who.int/tobacco/mpower/es/index.html Consultado el 2 de Agosto de 2009.

4.- American College of Physicians. Smoking Cessation. Ann Inter Med ITC 2007; 146: 2-16.

5.- AVEYARD P, WEST R. Managing smoking cessation. Br Med Journal 2007; 335: 37-41.

6.- LEMMENS V, OENEMA A, KNUT I K, BRUG J. Effectiveness of smoking cessation interventions among adults: a systematic review of reviews. European Journal of Cancer Prevention 2008; 17: 535-44.

7.- BELLO S, FLORES A, BELLO M. Tratamiento farmacológico del tabaquismo. Rev Chil Enf Respir 2008; 24: $127-37$.

8.- AMERICAN PSYCHIATRIC ASSOCIATION. Diagnostic and statistical manual of mental disorders. 4th ed., text revision: DSM-IV-TR. Washington, D.C.: American Psychiatric Association; 2000.

9.- WORLD HEALTH ORGANIZATION. International Classification of Diseases, 10th Edn. Geneva, World Health Organization, 1992.

10.- FIORE M C, JAÉN C R, BAKER T B, BAILEY W C, BENOWITZ N L, CURRY S J, et al. Treating Tobacco Use and Dependence: 2008 Update. Clinical Practice
Guideline. Rockville, MD: US Department of Health and Human Services, Public Health Service; 2008.

11.- FAGERSTRÖM KO. Measuring degree of physical dependence to tobacco smoking with reference to individualization of treatment. Addict Behav 1978; 3: 235-41.

12.- FAGERSTRÖM K, SCHEINEDER N. Measuring nicotine dependence. A review of the Fagerström tolerance questionaire. J Behav Med 1989; 12: 159-82.

13.- HEATHERTON T, KOZLOWSKI L, FRECKER R, FAGERSTRÖM K. The Fagerström test for nicotine dependence: a review of the Fagerström tolerance questionnaire. Br J Addiction 1991; 86: 1119-27.

14.- AMERICAN ACADEMY OF FAMILY PHYSICIANS. http://familydoctor.org:80/online/famdoces/home/ common/addictions/tobacco/296.html Consultado el 12 de Septiembre de 2009.

15.- MINISTERIO DE SALUD. Como ayudar a dejar de fumar. Manual para el equipo de salud. Programa de Salud Cardiovascular. 2003.

16.- LEWIS G, PELOSI A, ARAYA R, DUNN G. Measuring psychiatric disorder in the community: a standardized assessment for use by lay interviewers. Psychological Medicine 1992; 22: 465-86.

17.- HAN E, FOULDS J, STEINBERG M, GANDHI K K, WEST B, RICHARDSON D L, et al. Characteristics and smoking cessation outcomes of patients returning for repeat tobacco dependence treatment. Int J Clin Pract 2006; 60: 1068-74.

18.- SMITH S, JORENBY D, LEISCHOW S, NIDES M A, RENNARD S A, JOHNSTON J A, et al. Targeting smokers at increased risk for relapse: treating women and those with a history of depression. Nicotine Tob Res 2003; 5: 99-109.

19.- RICHMOND R L, KEHOE L, WEBSTER IW. Multivariate models for predicting abstention following intervention to stop smoking by general practitioner. Addiction 1993; 88: 1127-35.

20.- HURT R D. Treating Tobacco Dependence in a Medical Setting: Best Practices. Nicotine Dependence Center. Mayo Clinic, 2006.

21.- VEIGA S, MARTIN S, CORRAL L. Abordaje del tabaquismo: intervención mínima y herramientas psicológicas. Trastornos Adictivos 2003; 6: 95-102.

22.- RUSSELL M A, WILSON C, BAKER C D, TAYLOR C. Effect of general practitioners advise against smoking. Br Med J 1979; 2: 231-5.

23.- LANCASTER T, STEAD L F. Physician advice for smoking cessation (Cochrane Review). In: The Cochrane Library, Issue 4, 2005.

24.- LANCASTER T, STEAD L F. Individual behavioral counseling for smoking cessation (Cochrane Review). In: The Cochrane Library, Issue 4, 2004.

25.- FIORE M C, BAILEY W C, COHEN S J. Treating tobacco use and dependence: Clinical Practice Guideline. Interventions, nicotine replacement treatment, 
and several pharmacological treatments increase smoking cessation rates. Evidence-Based Mental Health 2001; 4: 16-24.

26.- SIMON J A, CARMODY T P, HUDES E S, SNYDER E, MURRAY J. Intensive smoking cessation counseling versus minimal counseling among hospitalized smokers treated with transdermal nicotine replacement: a randomized trial. Am J Med 2003; 114: 555-62.

27.- PROCHASKA J, DICLEMENTE C. Stages and processes of self-change of smoking: toward an integrative model of change. J Consult Clin Psychol 1983; 51: 390-95.

28.- PROCHASKA J O, GOLDSTEIN M G. Process of smoking cessation. Implications for clinicians. Clin Chest Med 1991; 12: 727-31.

29.- WEST R. Theory of Addiction. Wiley-Blackwell. 2006.

30.- WEST R. Theory of Motivation. http:/www.prime theory.com

31.- WEST R. Time for a change: putting the transtheoretical (stages of change) model to rest. Addiction 2005; 100: 1036-39.

32.- GLYNN T, MANLEY M. How to help your patients stop smoking. A National Cancer Institute Manual for Physicians. Bethestda MD. NIH Publication N ${ }^{\circ}$ 893064, 1990.

33.- U.S. PREVENTIVE SERVICES TASK FORCE. Counseling and Interventions to Prevent Tobacco Use and Tobacco-Caused Disease in Adults and Pregnant Women: U.S. Preventive Services Task Force Reaffirmation Recommendation Statement. Ann Intern Med 2009; 150: 551-5.

34.- ROYAL COLLEGE OF PHYSICIANS. Nicotine Addiction in Britain. A report of the Tobacco Advisory Group of the Royal College of Physicians. London: Royal College of Physicians, 2000.

35.- MINISTERIO DE SALUD Y AMBIENTE DE LA NACIÓN. Guía nacional de tratamiento de la adicción al tabaco de Argentina. Ministerio de Salud y Ambiente de la Nación. Buenos Aires, 2005.

36.- COMITÉ NACIONAL PARA LA PREVENCIÓN DEL TABAQUISMO (CNPT). Documento técnico de consenso sobre la atención sanitaria del tabaquismo en España. Observatorio para la prevención del Tabaquismo. Ministerio de Sanidad y Consumo. España, 2008.

37.- NATIONAL ADVISORY COMMITTEE ON HEALTH AND DISABILITY (NATIONAL HEALTH COMMITTEE) WELLINGTON, NEW ZEALAND. Guideline for smoking cessation. ISBN 0-478-25305-2 (Docu- ment) ISBN 0478-25306-0 (Internet) First Published in July 1999, Review date March 2004.

38.- RICE VH, STEAD LF. Nursing interventions for smoking cessation (Cochrane Review). In: The Cochrane Library, Issue 4, 2004. Oxford: Update Software.

39.- LANCASTER T, STEAD L F. Self-help interventions for smoking cessation. The Cochrane Database of Systematic Reviews 2005, Issue 3. CD001118.

40.- RUNDMO T, SMEDSLUND G, GOTESTAM K. Motivation for smoking cessation among the Norwegian public. Addict Behav 1997. 22: 377-86.

41.- ALBERG A, CARTER C, CARPENTER M. Weight gain as an impediment to cigarrette smoking cessation: a lingering problem in need of solutions. Prev Med 2007; 44: 296-7.

42.- CARPENTER M, HUGHES J, SOLOMON L, CALLAS P. Both smoking reduction with nicotine replacement therapy and motivational advice increase future cessation among smokers unmotivated to quit. J Consult Clin Psicol. 2004; 72: 371-81.

43.- CHAN S, LAM T, SALILI F, LEUNG G, WONG D, BOTHELO R, et al. A randomized controlled trial of an individualized motivational intervention on smoking cessation for parents of sick children: a pilot study. Appl Nurs Res 2005; 18: 178-81.

44.- BUTLER C, ROLLNICK S, COHEN D. Motivational consulting versus brief advice for smokers in general practice: a randomized trial. Br Gen Pract 1999; 49: 611-16.

45.- CAMARELLES F. Estrategias de intervención breve. Entrevista Motivacional en fumadores. En: JiménezRuiz CA, Fagerström KO (eds). Tratado de Tabaquismo, $2^{\circ}$ edición. Madrid. Ergon. 2007; 99-119.

46.- BELLO S, JARA N. Deja de Fumar ¡Ahora! Guía médica para dejar el tabaco. Ediciones B, Santiago de Chile. 2006.

47.- STEAD L F, LANCASTER T. Group behavior therapy programmes for smoking cessation. Cochrane Database of Systematic Reviews 2006.

48.- WEST R, MCNEILL A, RAW M. National smoking cessation guidelines for health professionals: an update. Thorax 2000; 55: 987-999.

49.- STEAD L F, LANCASTER T, PERERA R. Telephone counseling for smoking cessation. Cochrane Database of Systematic Reviews 2003; (4): CD002850.

50.- COLEMAN T, WILSON A. Factors associated with the provision of anti-smoking advice by general practitioners. Br J Gen Pract 1999; 49: 557-8.

\footnotetext{
Correspondencia a:

Dr. Sergio Bello S.

Instituto Nacional del Tórax

José Miguel Infante 717. Providencia

Santiago

Fono: 5754895

E-mail: sbello@torax.cl
} 